\title{
Cyclothymic Temperament is Associated with Poor Medication Adherence and Disordered Eating in Type 2 Diabetes Patients: A Case-Control Study
}

\author{
Tetsuya Yamamoto $\cdot$ Kenichi Sakurai · Masahiro Watanabe · \\ Ikki Sakuma · Nobuhisa Kanahara · Akihiro Shiina · Tadashi Hasegawa • \\ Hiroyuki Watanabe $\cdot$ Masaomi Iyo $\cdot$ Ryoichi Ishibashi (i)
}

Received: January 18, 2021 / Accepted: July 16, 2021 / Published online: July 31, 2021

(c) The Author(s) 2021

\section{ABSTRACT}

Introduction: Poor medication adherence and disordered eating are major self-care problems

Supplementary Information The online version contains supplementary material available at https:// doi.org/10.1007/s13300-021-01121-y.

T. Yamamoto $\cdot$ R. Ishibashi $(\bowtie)$

Division of Diabetes, Endocrinology and

Metabolism, Department of Medicine, Kimitsu

Chuo Hospital, Sakurai 1010, Kisarazu, Chiba 2920822, Japan

e-mail: ishibashi-cib@umin.net

T. Yamamoto $\cdot$ N. Kanahara $\cdot$ A. Shiina .

H. Watanabe

Center for Forensic Mental Health, Chiba

University, Chiba, Japan

K. Sakurai

Department of Nutrition and Metabolic Medicine, Center for Preventive Medical Sciences, Chiba

University, Chiba, Japan

M. Watanabe

Department of Sustainable Health Science, Center for Preventive Medical Sciences, Chiba University, Chiba, Japan

I. Sakuma · R. Ishibashi

Department of Endocrinology, Hematology and Gerontology, Chiba University Graduate School of Medicine, Chiba, Japan

N. Kanahara - A. Shiina - T. Hasegawa $\cdot$ M. Iyo Department of Psychiatry, Graduate School of Medicine and School of Medicine, Chiba University, Chiba, Japan in patients with type 2 diabetes that worsen glycemic control and increase the risk of developing severe diabetes complications. Affective temperament, which remains mostly unchanged throughout life, is speculated to predict poor treatment response and high comorbidity. The aim of this study was to explore the link between affective temperament and poor glycemic control due to insufficient self-care.

Methods: This single-center case-control study involved 77 outpatients divided into the 'poor glycemic control' group $(n=52)$ and the 'better glycemic control' group $(n=25)$ based on their mean glycated hemoglobin (HbA1c) levels over the past 12 months. All participants underwent one-on-one interviews during which they completed the following psychometric questionnaires: (1) the Mini-International Neuropsychiatric Interview 5.0.0; (2) the Temperament Evaluation of Memphis, Pisa, and San Diego Auto-questionnaire; (3) a researcherdesigned single question for assessing subclinical stress-induced overeating; and (4) the Morisky Medication Adherence Scale. The difference between two continuous independent variables was determined using Student's $t$ test. Discrete variables were compared using the Chi-square $\left(\chi^{2}\right)$ or Fisher's exact test. Multiple testing corrections were performed using the false discovery rate.

Results: Those outpatients in the poor glycemic control group exhibited significantly 
more stress-induced overeating $\left(\chi^{2}=1.14\right.$, $q$ statistic $=0.040)$ and poor medication adherence $(t=3.70, q=0.034)$ than those in the better glycemic control group. However, there were no significant differences between the two groups in terms of affective temperaments, clinical eating disorders, or diabetes-specific distress. Patients with stress-induced overeating $(t=-2.99, p=0.004)$ and poor medication adherence $(t=-4.34, p=0.000)$ exhibited significantly higher scores for cyclothymic temperament than their counterparts.

Conclusion: Cyclothymic temperament is significantly associated with disordered eating and/or poor medication adherence in patients with type 2 diabetes and is possibly linked to poor glycemic control.

Keywords: Affective temperament; Bipolar II disorders; Cyclothymic temperament; Depression; Disordered eating; Poor glycemic control; Poor medication adherence; Stressinduced overeating; TEMPS-A; Type 2 diabetes

\section{Key Summary Points}

Why carry out this study?

Poor medication adherence and disordered eating are major self-care problems in patients with type 2 diabetes (T2DM) because they worsen glycemic control, thereby increasing the risk of severe diabetes complications. On the contrary, affective temperament, which determines response to internal and external stress and remains mostly unchanged throughout life, can chronically influence self-care behaviors and glycemic control.

To date, there have been no published clinical studies aimed at comprehensively investigating the association between affective temperament, self-care problems, and poor glycemic control.
This study aimed to examine our hypothesis that affective temperament is a common cause of disordered eating and poor medication adherence and is thus placing these patients at risk of poor glycemic control.

\section{What was learned from the study?}

The 'poor glycemic' control group demonstrated significantly more stressinduced overeating $(\chi 2=1.14, q$ statistic $=$ $0.040)$ and poor medication adherence $(t=$ $3.70, q=0.034)$ than to those in the 'better control' group; however, there were no no significant differences between the two groups in terms of affective temperaments, clinical eating disorders, or T2DM-specific distress.

Cyclothymic temperament, a subtype of affective temperament, may be responsible for disordered eating and poor medication adherence in patients with T2DM and is possibly linked with poor glycemic control.

\section{INTRODUCTION}

The management of type 2 diabetes mellitus (T2DM) requires blood glucose levels to be maintained within the optimal glycemic range to avoid complications. However, long-term optimal glycemic control is difficult to maintain [1], and more than $30 \%$ of the patients with diabetes do not attain their individual glycemic goals [2]. Effective self-management of diabetes is essential to achieve optimal glycemic control and requires behavioral changes and/or adaptions [3].

The most common problems with self-management in patients with T2DM include poor medication adherence [2] and disordered eating [4], both of which result in poor glycemic control. Previous studies suggest that disordered eating affects up to $50 \%$ of the patients with T2DM [4] and is associated with increased 
retinopathy and neuropathy $[5,6]$ along with increased morbidity and mortality, high costs of outpatient care, and poor management of diabetes-associated complications [7].

Self-care behaviors and their subsequent medical outcomes in patients with T2DM are substantially influenced by complex individual, environmental, social, behavioral, and psychological factors [8]. While many studies have been conducted on the association between psychological factors and diabetes, including topics such as depression $[9,10]$, anxiety [11], chronic stress [12], anger [13], hostility [14], and diabetes-related distress [15], most have focused on the impact of these factors on diabetes onset, with only few exploring the psychological factors, such as temperament, which chronically influence self-care behaviors and glycemic control.

Temperament determines one's response to internal and external stimuli and remains mostly unchanged throughout life [16]. Therefore, problems with self-care behaviors attributed to temperament are difficult to change and can lead to long-term poor glycemic control. Affective temperament, conceptualized by Akiskal et al. [17] as characterized by depressive, hyperthymic, cyclothymic, irritable, and anxious types has gained the attention of researchers, and various studies have been conducted to associate these concepts with physical disease. One study reported that affective temperaments play a role in developing hypertension and arterial stiffening [18], whereas another study suggested that patients with T2DM and a depressive or anxious temperament exhibited poorer self-care behavior and metabolic control than patients without predominant affective temperaments [19].

To the best of our knowledge, no study has comprehensively analyzed the association of glycemic control, critical self-care behaviors, such as disordered eating and poor medication adherence, and affective temperaments. Therefore, the aim of this study was to clarify this association, employing structured interviews to differentiate clinically diagnosable affective disorders and eating disorders from subclinical statuses such as affective temperaments and disordered eating behaviors.

\section{METHODS}

\section{Ethics}

This study and its protocols were approved by the institutional review board of Chiba University School of Medicine (Reference No. 2175) and Kimitsu Chuo Hospital (Reference No. 272) in accordance to the Ethical Guidelines for Medical and Health Research Involving Human Subjects issued by the Ministry of Health, Labour and Welfare of Japan. The study was conducted in accordance with the Declaration of Helsinki 1964 and its later amendments; the Ethical Guidelines for Medical and Health Research Involving Human Subjects issued by the Ministry of Health, Labour and Welfare of Japan; the Clinical Trials Act; and other current legal regulations in Japan. Written informed consent was obtained from all participants after a providing them full explanation of this study, and all participants provided consent to participate. Authorization to use of MMAS-4 in this study was granted by Prof. DE Morisky, MMAS Research, LLC.

\section{Study Design and Recruitment Criteria}

This was a single-center case-control study. All patients with T2DM (aged 18-75 years) who had been attending the Department of Diabetes, Endocrinology, and Metabolism of Kimitsu Chuo Hospital for at least 3 years since September 2015 were screened for eligibility to participate in the study. The medical records of 905 patients with laboratory data from the 3 previous years and at least six glycated hemoglobin (HbA1c) measurements over the past 12 months were screened and relevant data extracted. Patients with a mean HbA1c $\geq 8.5 \%$ over the past 12 months (12mAv-HbA1c) were included in the 'poor glycemic control' group (PG), and those with $12 \mathrm{mAv}-\mathrm{HbA1c}$ levels $<$ $7.5 \%$ were included in the 'better glycemic control' group (BG). Patients with $12 \mathrm{mAvH}-$ bA1c levels between 7.5 and $8.5 \%$ were not included in the study since our aim was to focus on patients in the PG, a patient group that diabetologists have the most difficulty in 
helping achieve optimal glycemic control compared with patients with better glycemic control.

We estimated that the maximum number of one-on-one structured interviews we could conduct during the study period would be approximately 150; hence, we planned to recruit approximately 250 patients with T2DM to select the study participants. In the PG, we selected all 63 patients who suffered more than one diabetes complication as a predictive factor for long-term poor glycemic control, and among the remaining patients in the PG, we randomly selected 100 patients. Similarly, 100 patients of the BG were randomly selected. The purpose of the study was explained to all 263 patients. The exclusion criteria were: steroid therapy, dementia or schizophrenia, conditions that involve neurological manifestations (such as thyroid dysfunction, adrenal dysfunction, and collagen disease), hospital transfer, mortality, or discontinuation of treatment during the study period. A flowchart of the sampling procedure is shown in Fig. 1.

\section{Assessment of Clinical Characteristics}

Age, sex, duration of diabetes, history of psychiatric medication, and use of psychotropic medicines (antidepressants, mood stabilizers, and antipsychotics), some of which have adverse metabolic effects and worsen glycemic control, were assessed using a standardized questionnaire. We verified the use of insulin and/or glucagon-like peptide-1 (GLP-1) receptor agonist injection therapy and body mass index (BMI; $\mathrm{kg} / \mathrm{m}^{2}$ ) from medical charts.

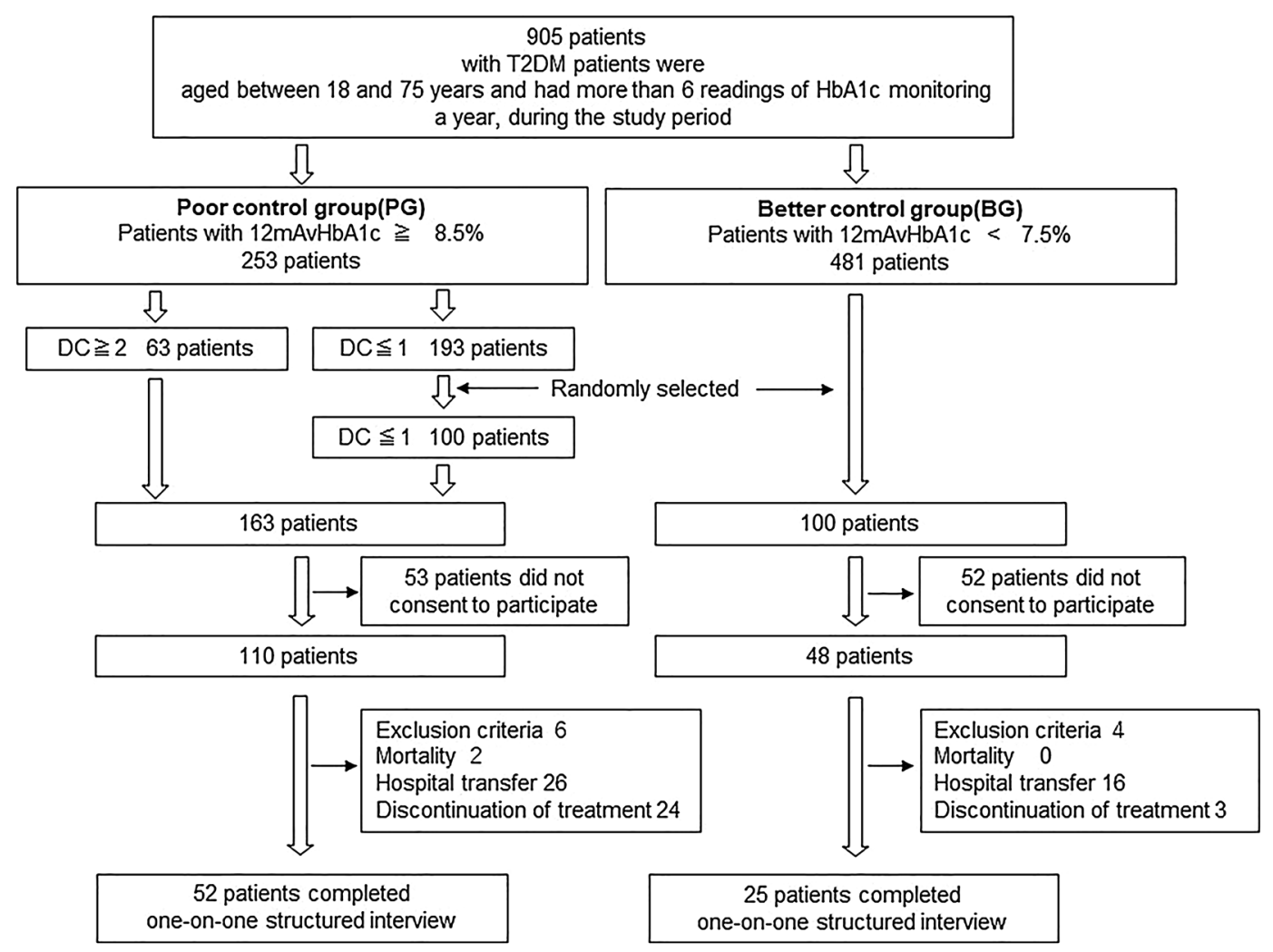

Fig. 1 Flowchart of patient sampling procedure. $D C$ Diabetes complication, including diabetic nephropathy, diabetic retinopathy, and diabetic neuropathy, $B G$ 'better the past 12 months, $H b A 1 c$ glycated hemoglobin, $P G$ 'poor glycemic control' group, $T 2 D M$ type 2 diabetes mellitus 


\section{Assessment of Psychological Conditions}

Bipolar I disorder, bipolar II disorder, major depressive disorder, bulimia nervosa, and binge eating disorder were diagnosed according to the Diagnostic and Statistical Manual of Mental Disorders (DSM-5), Fifth Edition (DSM-5; American Psychiatric Association, Washington DC, USA) and Mini-International Neuropsychiatric Interview 5.0.0 scores. Affective temperaments were evaluated using the full version of the Temperament Evaluation of Memphis, Pisa, and San Diego Auto-questionnaire (TEMPS-A) [20], which is a true-false self-reporting questionnaire comprising 110 questions that evaluate affective temperaments, including depressive, cyclothymic, hyperthymic, irritable, and anxious temperaments. The reliability and validity of the Japanese version of TEMPS-A have been established [21]. Temperament scores were converted into categorical variables with a cutoff point corresponding to a $z$-score of 1 , which is the minimum value that indicates excessive temperament. Regarding subclinical disordered eating, the researcher-designed question, "Have you experienced uncontrollable overeating as a reaction to a preceding stress-event or mood change?" was asked to confirm the presence or absence of stress-induced overeating [22] and/or mood instability. Medication adherence was evaluated using the Japanese translation of the 4-item Morisky Medication Adherence Scale (MMAS) [23-27]. ${ }^{1}$

\section{Statistical Analysis}

The difference between two continuous independent variables was determined using Student's $t$ test. Comparisons between two discrete variables were made using the chi-square test or Fisher's exact test. To evaluate the effects of affective temperaments on self-care behaviors, the TEMPS-A scores of each temperament were

\footnotetext{
1 (Note: The MMAS-4 Scale, content, name, and trademarks are protected by US copyright and trademark laws. Permission for use of the scale and its coding is required. A license agreement is available from MMAR, LLC., Donald E. Morisky, ScD, ScM, MSPH, 294 Lindura Ct., USA; donald.morisky@moriskyscalel.com.)
}

converted into $z$-scores before performing the Student's $t$ test, which was then used to compare the difference between the two stress-induced overeating groups and also between the two MMAS-4 groups. The two groups of each variable were defined as follows: stress-induced overeating was divided based on its existence (yes vs. no); MMAS-4 score was divided into two groups based on scores (better medication adherence $[\mathrm{BA}]=0$ or 1 vs. poor medication adherence $[\mathrm{PA}]=2-4$ ). The Japanese translation of the MMAS- 4 had not yet been validated at the time of the survey; therefore, we used these cutoff values because approximately $80 \%$ of the participants in our study had a score of 0 or 1 .

Multiple testing corrections were performed using the false discovery rate (Benjamini-Hochberg method), with appropriate $p$ values determined using two-tailed tests and $q$ values $<0.1$ considered to indicate significance. We constructed a path model using structural equation modeling (SEM), which posited that long-term glycemic control was associated with self-care behaviors and affective temperaments. Statistical analyses were performed using SPSS version 19 (SPSS-IBM, Armonk, NY, USA) and R (package version 3.6.1; $\mathrm{R}$ Foundation for Statistical Computing, Vienna, Austria).

\section{RESULTS}

\section{Participants}

In total, 263 patients with T2DM received an explanation on the purpose of the study, and 158 agreed to participate. Among these 158 patients, ten individuals who met the exclusion criteria were excluded from the interview, and 71 individuals (2 deaths, 42 hospital transfers, and 24 who discontinued treatment) dropped out from the study after initiating the survey. Ultimately, 52 patients in the PG group and 25 patients in the BG group completed the survey. The sampling procedures by group are shown in Fig. 1.

A comparison of the attributes and variables of participants in the PG and BG groups is presented in Table 1 . There was no significant 
Table 1 Demographic and medication variables according to mean glycated hemoglobin values over the past 12 months of $<7.5 \%$ (better glycemic control group) and $\geq 8.5 \%$ (poor glycemic control group)

\begin{tabular}{|c|c|c|c|}
\hline Variables & $\begin{array}{l}\text { Better glycemic control } \\
\text { group }(12 \mathrm{mAv}-\mathrm{HbA1c} \\
<7.5 \%), n=25\end{array}$ & $\begin{array}{l}\text { Poor glycemic control } \\
(12 \mathrm{mAv}-\mathrm{HbA1c} \\
\geq 8.5 \%), n=52\end{array}$ & Statistics \\
\hline Age, mean (SD) & $60.9(13.6)$ & $58.0(13.9)$ & $\begin{array}{l}t=0.9, p=0.39 \\
q=0.737\end{array}$ \\
\hline Female, \% & 52.0 & 40.0 & $\begin{array}{l}\chi^{2}=0.895, p=0.81 \\
q=1.000\end{array}$ \\
\hline $\begin{array}{c}\text { Duration of diabetes, } \\
\text { years, mean }(\mathrm{SD})\end{array}$ & $14.5(9.5)$ & $17.1(10.0)$ & $\begin{array}{l}t=1.1, p=0.28 \\
q=0.595\end{array}$ \\
\hline BMI, mean (SD) & $27.3(4.1)$ & $27.2(5.1)$ & $\begin{array}{l}t=0.1, p=0.89 \\
q=1.000\end{array}$ \\
\hline Use of injection therapy ${ }^{\mathrm{a}}, \%$ & 34.7 & 71.4 & $\begin{array}{l}\chi^{2}=9.49, p=0.003 \\
q=0.034^{*}\end{array}$ \\
\hline History of psychiatric medication, $\%$ & 24.0 & 7.7 & $\begin{array}{l}\chi^{2}=2.12, p=0.07 \\
q=0.300\end{array}$ \\
\hline Use of antidepressants, \% & 0 & 0 & - \\
\hline Use of antipsychotics, \% & 0 & 0 & - \\
\hline
\end{tabular}

$12 m A v-H b A l c$ Mean HbAlc over the past 12 months, BMI body mass index, FDR false discovery rate, $q q$ statistic, $S D$ standard deviation

${ }^{*} q<0.1$

${ }^{a}$ Injection therapy includes insulin and glucagon-like peptide-1 analogs

difference between the two groups in terms of age, sex, duration of disease, and current BMI. The use of insulin and/or GLP-1 receptor agonist injection therapy was significantly higher in the PG group than in the BG group (34.7 vs. $71.4 \%, q=0.034)$.

\section{Glycemic Control and Self-care Behaviors}

Regarding self-care behaviors, patients in the PG group exhibited significantly higher stress-induced overeating $\left(\chi^{2}=1.14, q=0.040\right)$ and poorer medication adherence $(t=3.7$, $q=0.034)$ than those in the BG group. However, there was no significant difference between the groups in terms of clinical eating disorders.

\section{Psychiatric/Psychological Factors in the PG and BG groups}

There was no significant difference in both the history of psychiatric medication and the use of psychotropic medicines, such as antidepressants, mood stabilizers, and antipsychotics, between the two groups. No patient was diagnosed with bipolar I disorder according to Table 2 in either group. Although several psychological conditions were identified in both groups, there was no significant difference between the patients in the BG and PG groups in terms of prevalence of bipolar II disorder according to Table 2 (4.5 vs. $7.5 \%, q=1.000$ ), major depressive disorder (18.2 vs. $7.5 \%$, $q=1.000)$, bulimia nervosa (0.0 vs. $5.4 \%$, 
$q=0.819)$, and binge eating disorder (0.0 vs. $10.5 \%, q=0.819)$.

\section{Affective Temperament in the PG and BG groups}

In this study, there was no significant difference between the $B G$ and $P G$ groups regarding the following predominant affective temperaments: depressive ( 9.1 vs. $25.6 \%, q=0.408$ ), cyclothymic ( 13.6 vs. $15.3 \%, q=1.000)$, hyperthymic (9.1 vs. $20.5 \%, q=0.595)$, irritable (13.6 vs. $17.9 \%, q=0.935)$, and anxious (27.3 vs. $15.3 \%$, $q=0.595)$.

\section{Association Between Self-care Behaviors and Affective Temperaments}

The group with stress-induced overeating showed a higher cyclothymic temperament score than did the group without stress-induced overeating (z-score: 0.391 vs. -0.332 , $p=0.004)$; however, there were no differences in other temperaments between the groups (Fig. 2). In addition, patients categorized in the group with poor medication adherence had significantly higher scores for cyclothymic temperament (z-score: 1.133 vs. -0.227 , $p=0.000)$ than those categorized in the group with better medical adherence, while other temperaments did not differ between the groups (Fig. 3).

\section{Association Between Injection Therapy and Self-care Behaviors}

Poor self-care behaviors, such as disordered eating and poor medication adherence, are considered to be associated with diabetes injection therapy $[28,29]$. However, in our study, there was no significant association between these behaviors and injection therapy, as shown in Electronic Supplementary Material Table S1.

\section{Relationship between Affective Temperaments, Self-care Behaviors, and Glycemic Control}

Path analysis using SEM was performed to evaluate the hypothetical pathway from cyclothymic temperament that was significantly associated with disordered eating and poor medication adherence in our analysis of glycemic control through self-care behaviors. The model shown in Fig. 4 accurately illustrates the data according to all goodness-of-fit measures: model $\chi^{2}=0.5444, d f=3, p=0.909$, Akaike information criterion $(\mathrm{AIC})=14.544$, and Bayesian information criterion $(B I C)=-11.478$. The path coefficients of cyclothymic temperament toward stress-induced overeating $(0.449, p<0.001)$ and medication adherence $(0.498, p<0.001)$, as well as that of medication adherence toward $12 \mathrm{mAv}$ HbA1c $(0.408, p<0.001)$, were highly significant. The path coefficient of stress-induced overeating toward $12 \mathrm{mAv}-\mathrm{HbA1c}$ was not significant $(0.228, p=0.056)$ but showed a potential association.

\section{DISCUSSION}

In this study we tested the hypothesis that disordered eating and poor medication adherence, which are regarded as critical self-care behaviors that influence glycemic control, may be significantly affected by emotional factors. In particular, affective temperament could chronically affect these self-care behaviors, resulting in poor long-term glycemic control. The results suggest that in our patient population: (1) disordered eating and poor medication adherence were significantly associated with poor glycemic control; and (2) cyclothymic temperament-a type of affective temperament-was significantly associated with disordered eating and PA; in addition, (3) the SEM analysis indicated that there is a possible causal linkage between cyclothymic temperament and poor glycemic control modulated by disordered eating and poor medication adherence.

Individuals with cyclothymic temperaments begin to show clinical characteristics from their 
Table 2 Psychological and self-care behavior variables according to $12 \mathrm{mAv}-\mathrm{HbAlc}$ values $<7.5 \%$ (better glycemic control group) and $\geq 8.5 \%$ (poor glycemic control group)

\begin{tabular}{|c|c|c|c|}
\hline Variables & $\begin{array}{l}\text { Better glycemic control group } \\
(12 \mathrm{mAv}-\mathrm{HbA1c} \\
<7.5 \%), n=25\end{array}$ & $\begin{array}{l}\text { Poor glycemic control group } \\
\text { (12mAv-HbA1c } \\
\geq 8.5 \%), n=52\end{array}$ & Statistics \\
\hline Bipolar I disorder, \% & 0.0 & 0.0 & - \\
\hline Bipolar II disorder, \% & 4.5 & 7.5 & $\begin{array}{l}\chi^{2}=0.20, p=1.00 \\
q=1.000\end{array}$ \\
\hline $\begin{array}{l}\text { Major depressive } \\
\text { disorder, } \%\end{array}$ & 18.2 & 7.5 & $\begin{array}{l}\chi^{2}=0.20, p=1.00 \\
q=1.000\end{array}$ \\
\hline $\begin{array}{l}\text { Depressive } \\
\text { temperament }{ }^{\mathrm{a}}, \%\end{array}$ & 9.1 & 25.6 & $\begin{array}{l}\chi^{2}=2.40, p=0.12 \\
q=0.408\end{array}$ \\
\hline $\begin{array}{l}\text { Cyclothymic } \\
\text { temperament }^{\mathrm{a}}, \%\end{array}$ & 13.6 & 15.3 & $\begin{array}{l}\chi^{2}=0.93, p=0.85 \\
q=1.000\end{array}$ \\
\hline $\begin{array}{l}\text { Hyperthymic } \\
\text { temperament }^{\mathrm{a}}, \%\end{array}$ & 9.1 & 20.5 & $\begin{array}{l}\chi^{2}=1.34, p=0.25 \\
q=0.595\end{array}$ \\
\hline $\begin{array}{l}\text { Irritable temperament }{ }^{\mathrm{a}} \text {, } \\
\%\end{array}$ & 13.6 & 17.9 & $\begin{array}{l}\chi^{2}=0.19, p=0.66 \\
q=0.935\end{array}$ \\
\hline $\begin{array}{l}\text { Anxious temperament }{ }^{\mathrm{a}} \text {, } \\
\%\end{array}$ & 27.3 & 15.3 & $\begin{array}{l}\chi^{2}=1.26, p=0.26 \\
q=0.595\end{array}$ \\
\hline Bulimia nervosa, \% & 0.0 & 5.4 & $\begin{array}{l}\chi^{2}=1.18, p=0.53 \\
q=0.819\end{array}$ \\
\hline Binge eating disorder, \% & 0.0 & 10.5 & $\begin{array}{c}\chi^{2}=102.4, p=0.53 \\
q=0.819\end{array}$ \\
\hline $\begin{array}{l}\text { Stress-induced } \\
\text { overeating, yes, \% }\end{array}$ & 22.8 & 60.0 & $\begin{array}{c}\chi^{2}=1.14, p=0.007 \\
q=0.040^{*}\end{array}$ \\
\hline $\begin{array}{l}\text { MMAS-4 score, mean } \\
\text { (SD) }\end{array}$ & $1.3(1.2)$ & $3.4(3.1)$ & $\begin{array}{l}t=3.7, p=0.004 \\
q=0.034^{*}\end{array}$ \\
\hline
\end{tabular}

MMAS-4 Morisky Medication Adherence Scale

${ }^{*} q<0.1$

a Temperament Evaluation of Memphis, Pisa, and San Diego Auto-questionnaire (TEMPS-A)

teens into early adulthood. Such individuals experience both manic and depressive phases, such as lassitude and physical discomfort versus feeling good and having pessimistic thoughts versus a tendency for overoptimism accompanied by a lack of concern, all of which can potentially lead to painful consequences. Generally, each phase lasts only a few days, contributing to a relatively unstable mood [17].

Several studies have demonstrated a positive association between cyclothymic temperament and eating disorders. Ramacciotti et al. 


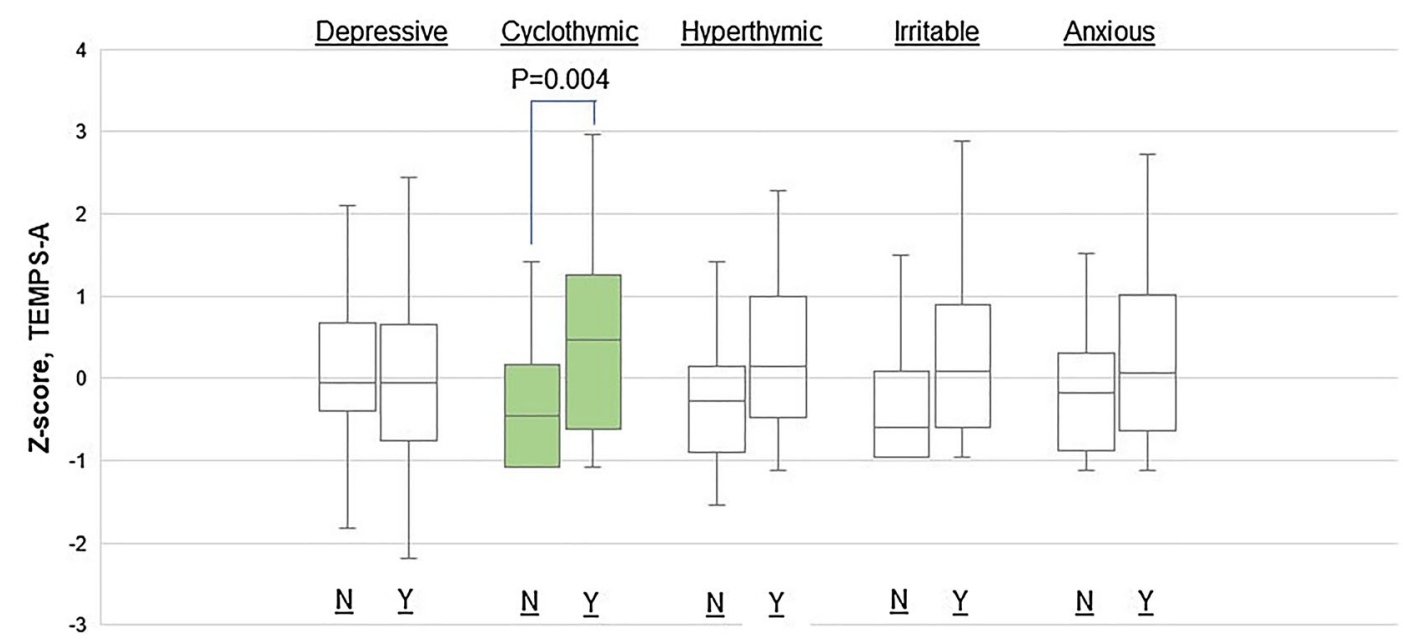

Fig. 2 Box plot of presence of stress-induced overeating. Stress-induced overeating can be seen shown to have a significant relationship with cyclothymic temperament (boxplot in green). Scores of the Temperament Evaluation of Memphis, Pisa, and San Diego Auto-questionnaire
(TEMPS- $A$ ) were converted into $z$-scores, and the Student's $t$ test was performed using the averages of $z$ scores for comparing groups according to the presence (yes $[Y]$ ) or absence (no $[N]$ ) of stress-induced overeating

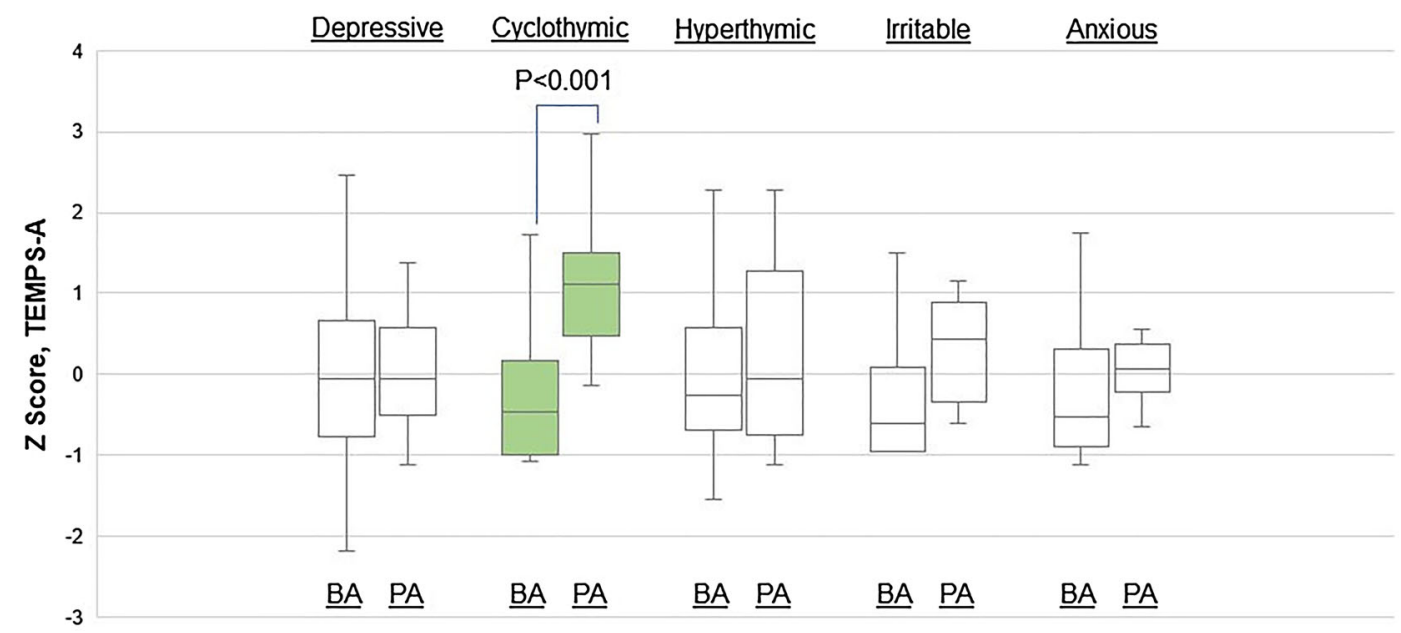

Fig. 3 Box plot of TEMPS-A scores between the better medication adherence $(B A)$ group and the poor medication adherence $(P A)$ group. Poor medical adherence can be seen to have a significant relationship with cyclothymic temperament (boxplot in green). TEMPS-A scores were

investigated the affective temperaments in a sample of patients with eating disorders and identified that only cyclothymic temperament was significantly higher in the binge eating subgroup [30]. D'Ambrosio et al. investigated converted into $z$-scores, and the Student's $t$ test was performed using the averages of $z$-scores to compare better the BA group (score $=0$ or 1 ) with the PA group (score $=2$ to 4 ), categorized by the Morisky Medication Adherence Scale (MMAS-4) score

affective temperaments and clinical characteristics in patients with obsessive/compulsive disorders and found that patients with cyclothymic temperament showed significantly more repeating compulsions and higher 


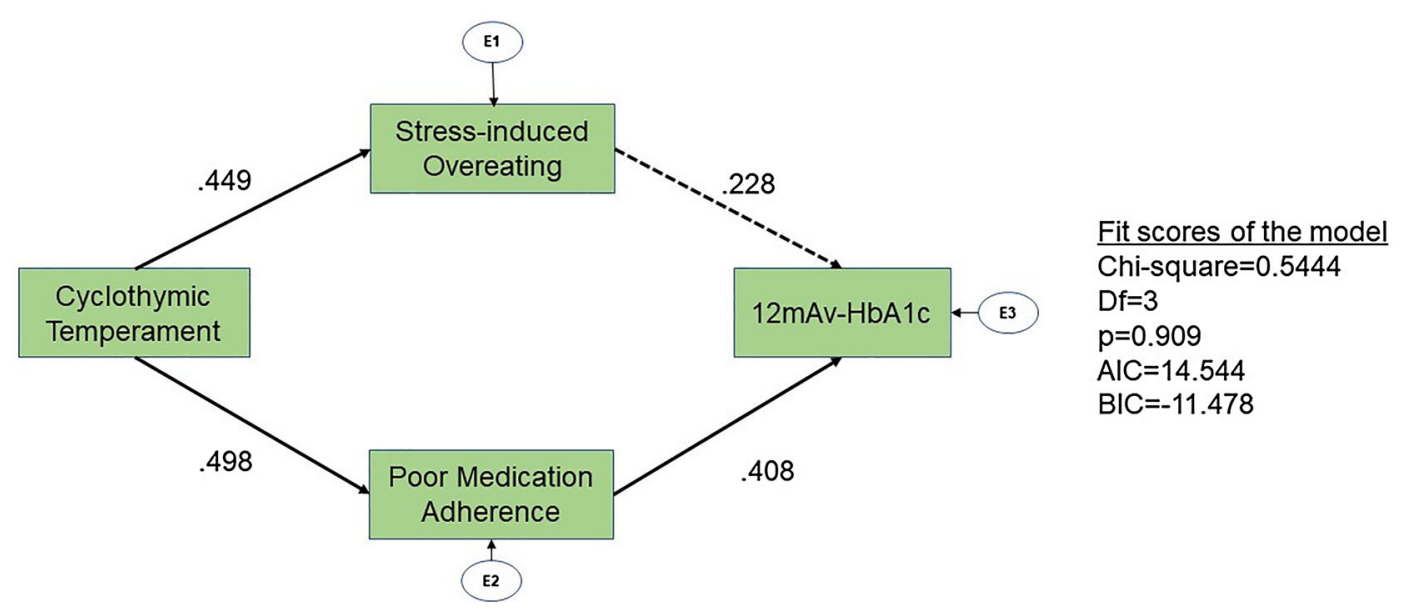

Fig. 4 Structure equation model with $12 \mathrm{mAv}-\mathrm{HbA} \mathrm{c}$, stress-induced overeating, MMAS- 4 score, and cyclothymic temperament as per the TEMPS-A score. The arrows with solid lines represent significant pathways, and the arrow

comorbidity rates with eating disorders than did other patient groups [31].

As a background of these associations, the following psychopathological process is assumed. People with cyclothymic temperament are impulsive and prone to addiction to control mood instability [32]. Psychological stress associated with mood instability may predispose patients to stress-induced overeating, with acceleration in the consumption of energy- and nutrient-dense processed foods that have high sugar and fat content [33] and considered to be addictive substances [34]. Specifically, instead of dealing with mood instability appropriately, these people tend to engage in "dysfunctional mood modulatory behaviors," such as overeating, which may reduce awareness of mood status and neutralize the distress of mood change [35]. Continuous overeating of nutrient-dense processed foods may pose addictive and long-lasting disordered eating. Additionally, the constitutional disposition of overoptimism and risk-taking represented in cyclothymic temperament may influence the long-term course of refined food addiction $[32,34]$ and poor medication adherence. Although our study has not investigated the chronicity of disordered eating and poor medication adherence, symptomatic traits of cyclothymic temperaments, such as mood with the broken line represents a non-significant pathway. The number beside the arrows indicates the standardized path coefficient (minimum - 1, maximum 1).E1,E2, E3 Error variables

instability, impulsivity, and overoptimism, may induce or prolong disordered eating and/or poor medication adherence and subsequently lead to poor glycemic control.

In contrast, it is also essential for clinicians to know that patients with cyclothymic temperament are possibly in the subclinical phase of bipolar disorder or may develop bipolar disorder in the future $[17,36-38]$. In addition to cyclothymic temperament, we identified patients with undiagnosed bipolar II disorder in this study. Individuals with bipolar II disorder may show only depressive symptoms in the subclinical phase; however, indiscriminate use of antidepressants may increase agitation risk in these patients [39]. Therefore, this psychological state requires the attention of clinicians.

In contrast, however, we previously reported a case [40] in which never-diagnosed bipolar II disorder was identified in a patient with T2DM with a chronic eating disorder and poor glycemic control. Treatment with a mood-stabilizer resulted in a remarkable improvement in both the eating disorder and glycemic control.

In summary, identifying cyclothymic temperament in patients with T2DM will allow clinicians to address long-term disordered eating, poor medication adherence, and poor glycemic control. Furthermore, referral of patients with severe symptoms of cyclothymic 
temperament to a mental health professional may increase their chances to improve self-care behaviors and glycemic control with appropriate medication in case the patient has a comorbid affective disorder.

To assess cyclothymic and other temperaments, TEMPS-A short-version [41], which comprises 39 items instead of the 110 items in the full version), may have better usability. Moreover, further development of more straightforward and usable questionnaires that could distinguish cyclothymic temperament in patients with T2DM is required. This may include a combination of TEMPS-A and questionnaires to assess disordered eating and poor medication adherence.

This study has several strengths. First, both poor medication adherence and disordered eating, which are two key risk factors for poor glycemic control that are commonly observed in clinical settings, were investigated. Second, we used a standardized structured interview to distinguish diagnosable affective/eating disorders, subthreshold affective conditions, and disordered eating. Third, we used the $12 \mathrm{mAv}$ HbA1c, which reflects the status of the yearlong self-care management.

This study also has several limitations. First, this was a single-center study with small sample size. We did not perform a power analysis at the beginning of the study because it was challenging to formulate the sample design owing to the lack of previous studies with similar objectives. Hence, we considered the current study to be a pilot study and conducted one-onone structured interviews, which, in effect, limited the total sample size due to the timeconsuming nature of this type of interview. Since our study's sample size was small, the results may not adequately reflect the difference in affective temperament between the PG and BG. Nevertheless, our findings may be used as a basis for larger studies. Second, the general population was not included. We also did not establish the validity and reliability of the researcher-made questionnaire. Future studies should be performed with larger samples covering the general population and more detailed and reliable indices of eating behaviors.

\section{CONCLUSIONS}

Cyclothymic temperament is significantly associated with disordered eating and poor medication adherence, which are major causes of poor glycemic control among patients with T2DM. Our findings can potentially convince clinicians of the necessity of increasing their awareness of the cyclothymic temperament and its relationship with T2DM and affective disorders. This awareness may contribute towards the patient achieving optimal glycemic control and receiving appropriate psychiatric treatment if necessary.

\section{ACKNOWLEDGEMENTS}

The authors thank all study participants and all clinical staff members for their assistance in the execution of the study.

Funding. No funding or sponsorship was received for this study or for publishing this article. The Rapid Service Fee was funded by the authors.

Editorial and Other Assistance. The authors thank Atsuko Watanabe, Tetsushi Takeda, and Chiho Ito for their valuable assistance in preparing the manuscript. We thank Editage (www.Editage.com) for English language editing. The editing service fee was funded by the authors.

Authorship. Tetsuya Yamamoto, Kenichi Sakurai, Masahiro Watanabe, Ikki Sakuma, Nobuhisa Kanahara, Akihiro Shiina, Tadashi Hasegawa, Hiroyuki Watanabe, Masaomi Iyo, Ryoichi Ishibashi met the International Committee of Medical Journal Editors criteria for authorship for this article, take responsibility for the integrity of the work as a whole, and have given their approval for this version to be published.

Authors' Contributions. Tetsuya Yamamoto developed the concept of this research, and Tetsuya Yamamoto, Kenichi 
Sakurai, Masahiro Watanabe, Ikki Sakuma, Nobuhisa Kanahara, Akihiro Shiina, Tadashi Hasegawa, Hiroyuki Watanabe, Masaomi Iyo, Ryoichi Ishibashi designed the study. Tetsuya Yamamoto researched the data. Masahiro Watanabe was in charge of the statistical analysis. Tetsuya Yamamoto, Kenichi Sakurai, Masahiro Watanabe, Ikki Sakuma, Nobuhisa Kanahara, Akihiro Shiina, Tadashi Hasegawa, Hiroyuki Watanabe, Masaomi Iyo, and Ryoichi Ishibashi wrote the manuscript.

Prior Presentation. The results of this study were partly disseminated at the 60th Annual Meeting of the Japan Diabetes Society on 18 May 2017, in Nagoya, Japan.

Disclosures. Ryoichi Ishibashi has received research funds from Astellas Pharma Inc., Amgen Astellas BioPharma K.K., Daiichi Sankyo Co. Ltd., Taisho Pharmaceutical Co. Ltd., Takeda Pharmaceutical Co., Ltd., MSD K.K., Boehringer Ingelheim Pharmaceuticals, Inc., and Mitsubishi Tanabe Pharma Corporation. Tetsuya Yamamoto, Kenichi Sakurai, Masahiro Watanabe, Ikki Sakuma, Nobuhisa Kanahara, Akihiro Shiina, Tadashi Hasegawa, Hiroyuki Watanabe, and Masaomi Iyo declare that they have no conflict of interest.

Compliance with Ethics Guidelines. This study and its protocols were approved by the institutional review board of Chiba University School of Medicine (Reference No. 2175) and Kimitsu Chuo Hospital (Reference No. 272) in accordance to the Ethical Guidelines for Medical and Health Research Involving Human Subjects issued by the Ministry of Health, Labour and Welfare of Japan. The study was conducted in accordance with the Declaration of Helsinki 1964 and its later amendments; the Ethical Guidelines for Medical and Health Research Involving Human Subjects issued by the Ministry of Health, Labour and Welfare of Japan; the Clinical Trials Act; and other current legal regulations in Japan. Written informed consent was obtained from all participants after a providing them full explanation of this study, and all participants provided consent to participate. Authorization to use of MMAS- 4 in this study was granted by Prof. DE Morisky, MMAS Research, LLC, 2020 Glencoe Ave, CA 90,291-4007 (moc.liamg@yksiromd).

Data Availability. The datasets generated during and/or analyzed during the current study are not publicly available owing to the lack of approval for data sharing from the institutional review board of Kimitsu Chuo Hospital.

Open Access. This article is licensed under a Creative Commons Attribution-NonCommercial 4.0 International License, which permits any non-commercial use, sharing, adaptation, distribution and reproduction in any medium or format, as long as you give appropriate credit to the original author(s) and the source, provide a link to the Creative Commons licence, and indicate if changes were made. The images or other third party material in this article are included in the article's Creative Commons licence, unless indicated otherwise in a credit line to the material. If material is not included in the article's Creative Commons licence and your intended use is not permitted by statutory regulation or exceeds the permitted use, you will need to obtain permission directly from the copyright holder. To view a copy of this licence, visit http://creativecommons.org/licenses/by$\mathrm{nc} / 4.0 /$.

\section{REFERENCES}

1. Wallance TM, Matthews DR. Poor glycaemic control in type 2 diabetes, conspiracy of disease, suboptimal therapy and attitude. QJM. 2000;93: 369-74.

2. Edelman SV, Polonsky WH. Type 2 diabetes in the real world: the elusive nature of glycemic control. Diabetes Care. 2017;40:1425-32.

3. Reyes J, Tripp-Reimer T, Parker E, Muller B, Laroche $H$. Factors influencing diabetes self-management among medically underserved patients with Type II diabetes. Glob Qual Nurs Res. 2017;4: 2333393617713097.

4. Nip ASY, Reboussin BA, Dabelea D, et al. Disordered eating behaviors in youth and young adults with 
type 1 or type 2 diabetes receiving insulin therapy: the SEARCH for diabetes in youth study. Diabetes Care. 2019;42:859-66.

5. Rydall AC, Rodin GM, Olmsted MP, Devenyi RG, Daneman D. Disordered eating behavior and microvascular complications in young women with insulin-dependent diabetes mellitus. N Engl J Med. 1997;336:1849-54.

6. Steel JM, Young RJ, Lloyd GG, Clarke BF. Clinically apparent eating disorders in young diabetic women: associations with painful neuropathy and other complications. Br Med J (Clin Res Ed). 1987;294:859-62.

7. Zoungas S, Chalmers J, Ninomiya T, et al. Association of HbA1c levels with vascular complications and death in patients with type 2 diabetes: evidence of glycaemic thresholds. Diabetologia. 2012;55: 636-43.

8. Young-Hyman D, de Groot M, Hill-Briggs F, et al. Psychosocial care for people with diabetes: a position statement of the American Diabetes Association. Diabetes Care. 2016;39:2126-40.

9. Knol MJ, Twisk JW, Beekman AT, et al. Depression as a risk factor for the onset of type 2 diabetes mellitus. A meta-analysis. Diabetologia. 2006;49: $837-45$.

10. Mezek B, Eaton WW, Albrecht S, et al. Depression and type 2 diabetes over the lifespan: a meta-analysis. Diabetes Care. 2008;31:2383-90.

11. Engum A. The role of depression and anxiety in onset diabetes in a large population-based study. J Psychosom Res. 2007;62:31-8.

12. Kumari M, Head J, Marmot M. Prospective study of social and other risk factors for incidence of type 2 diabetes in the Whitehall II study. Arch Intern Med. 2004;164:1873-80.

13. Golden SH, William JE, Ford DE, et al. Anger temperament in modestly associated with the risk of type 2 diabetes mellitus. The Atherosclerosis Risk in Communities Study. Psychoneuroendocrinology. 2006;31:325-32.

14. Bunde J, Suls J. A quantitative analysis of the relationship between the Cook-Medley Hostility Acale and traditional coronary artery disease risk factors. Health Psychol. 2006;25:493-500.

15. Polonsky WH, Anderson BJ, Lohrer PA, et al. Assessment of diabetes-related distress. Diabetes Care. 1995;18:754-60.
16. Russo M, Mahon K, Shanahan M, et al. Affective temperaments and neurocognitive functioning in bipolar disorder. J Affect Disord. 2014;169:51-6.

17. Vazquez GH, Gonda X. Affective temperaments and mood disorders: a review of current knowledge. Curr Psychiatry Rev. 2013;9:21-32.

18. László A, Tabák Á, Kőrösi B, et al. Association of affective temperaments with blood pressure and arterial stiffness in hypertensive patients: a crosssectional study. Cardiovasc Disord. 2016;16:158.

19. Gois C, Barbosa A, Ferro A, et al. The role of affective temperaments in metabolic control in patients with type 2 diabetes. J Affect Disord. 2011;134: $52-8$.

20. Akiskal HS, Akiskal KK, Haykal RF, Manning JS, Connor PD. TEMPS-A: progress towards validation of self-rated clinical version of the Temperament Evaluation of the Memphis, Pisa, Paris, and San Diego Autoquestionnaire. J Affect Disord. 2005;85: 3-16.

21. Matsumoto S, Akiyama T, Tsuda H, et al. Reliability and validity of TEMPS-A in a Japanese non-clinical population: application to unipolar and bipolar depressives. J Affect Disord. 2005;85:85-92.

22. Wallis DJ, Hetherington MM. Stress and eating: the effects of ego-threat and cognitive demand on food intake in restrained and emotional eaters. Appetite. 2004;43:39-46.

23. Kamishima S, Noji R, Katakura Y, Maruyama T. Factors related to adherence to a medication regimen in out-patients being treated for stroke. J Jpn Acad Nurs Sci. 2008;28(1):21-30 (in Japanese).

24. Sakurai H, Onda M, Norose T. An empirical study on the influence structure of medication adherence: Comparative model analysis between diabetic and hypertensive patients. J Pharm Commun. 2017;15(2):4-1 (in Japanese).

25. Morisky DE, Green LW, Levine DM. Concurrent and predictive validity of a self-reported measure of medication adherence. Med Care. 1986;24:67-74.

26. Morisky DE, Malotte CK, Choi P, et al. A patient education program to improve rate with antituberculosis drug regimens. Health Educ Q. 1990;17: 253-68.

27. Morisky DE, DiMatteo MR. Improving the measurement of self-reported medication nonadherence: Final response. J Clin Epidemiol. 2011;64(3): 262-4.

28. Lewey J, Wei W, Lauffenburger JC, et al. Targeted adherence intervention to reach glycemic control 
with insulin therapy for patients with diabetes (TARGIT-diabetes): rationale and design of a pragmatic randomised clinical trial. BMJ Open. 2017;7: e016551.

29. Hartman I. Insulin analogs: impact on treatment success, satisfaction, quality of life, and adherence. Clin Med Res. 2008;6:54-67.

30. Ramacciotti CE, Paoli RA, Ciapparelli A, et al. Affective temperament in the eating disorders. Eat Weight Disord. 2004;9:114-9.

31. D'Ambrosio V, Albert U, Bogetto F, Maina G. Obsessive-compulsive disorder and cyclothymic temperament: an exploration of clinical features. J Affect Disord. 2010;127:295-9.

32. Maremmani I, Perugi G, Pacini M, Akiskal HS. Toward a unitary perspective on the bipolar spectrum and substance abuse: opiate addiction as a paradigm. J Affect Disord. 2006;93:1-12.

33. Cameron MJ, Maguire RW, McCormack J. Stressinduced binge eating: A behavior analytic approach to assessment and intervention. J Adult Dev. 2011;18:81-4.

34. Ifland JR, Preuss HG, Marcus MT, et al. Refined food addiction: a classic substance use disorder. Med Нypo. 2009;72:518-26.

35. Cooper Z, Fairburn CG. The evolution of "enhanced" cognitive behavior therapy for eating disorders: learning from treatment nonresponse. Cogn Behav Pract. 2011;18:394-402.

36. Judd LL, Akiskal H, Schetter PJ, et al. A prospective investigation of the natural history of the long-term weekly symptomatic status of bipolar II disorder. Arch Gen Psychiatry. 2003;60:261-9.

37. Kahler CW, Spillane NS, Metrik J, Leventhal AM, Monti PM. Sensation seeking as a predictor of treatment compliance and smoking cessation treatment outcomes in heavy social drinkers. Pharmacol Biochem Behav. 2009;93:285-90.

38. Gassab L, Mechri A, Bacha M, Gaddour N, Gaha L. Affective temperaments in the bipolar and unipolar disorders: distinctive profiles and relationship with clinical features. Encephale. 2008;34:477-82 (in French).

39. Baldessarini RJ, Vázquez GH, Tondo L. Bipolar depression: a major unsolved challenge. Int $\mathrm{J}$ Bipolar Disord. 2020;8:1.

40. Yamamoto T, Kanahara N, Hirai A, Watanabe H, Iyo $\mathrm{M}$. Lamotrigine in binge-eating disorder associated with bipolar II depression and treatment-resistant type 2 diabetes mellitus: a case report. Clin Neuropharmacol. 2013;36:34-5.

41. Nakato $Y$, Inoue $T$, Nakagawa $S$, et al. Confirmation of the factorial structure of the Japanese short version of the TEMPS-A in psychiatric patients and general adults. Neuropsychiatr Dis Treat. 2016;12: 2173-9. 